\title{
A new large projection operator for the redundancy framework
}

\author{
Mohammed Marey \& François Chaumette
}

\begin{abstract}
In this paper, we propose a new projection operator for the redundancy framework based on a task function defined as the norm of the usual error. This projection operator allows performing secondary tasks even when the main task is full rank. To ensure the convergence of the system, a switching strategy is then defined to switch from the new projection operator to the classical one before the norm of the total error reaches zero. An adaptive gain is also defined to slow down the convergence of the main task. It allows the secondary tasks to be active for longer. The experimental results obtained show the agreement with the analytical study and demonstrate the effectiveness of the proposed projection operator with respect to the classical one.

Index Terms-Robot redundancy, secondary task, projection operator, visual servoing.
\end{abstract}

\section{INTRODUCTION}

Redundancy allows robot systems to increase their level of manipulability and flexibility. There are several types of redundancy for a robot manipulator: redundancy with respect to the end effector when the manipulator has more DOFs than those required to place its end effector at a given position and orientation within the task space, and redundancy with respect to the task when the number of independent parameters required by a task is less than the DOFs of the robot manipulator [5],[4],[13],[3]. Several works have been done on the utilization of redundancy to consider different sorts of constraints. This is usually done by combining secondary tasks that express these constraints with the main task [1],[6],[2],[7],[12],[9].

In [1], a global objective function that realizes a compromise between the main task and secondary tasks is used by exploiting the robot redundant DOFs with respect to the main task. This approach was used to avoid kinematic singularities and joint limits in a target tracking system. However, important perturbations can be produced by the obtained motions, which are generally not compatible with the regulation to zero of the main task. Also, the global task can fail when the same joints are used for the avoidance and for achieving the main task. Another classical approach uses the gradient projection method [10]. This is done by projecting any vector representing the desired motion in the space of the secondary task onto the null space of the Jacobian of the main task to modify the behavior of the system, but not the convergence of the main task. This method requires that the main task does not constrain all the robot DOFs. Indeed, in that case, the main task Jacobian

Mohammed Marey and François Chaumette are with INRIA, Centre Rennes-Bretagne Atlantique, IRISA, Campus de Beaulieu, F35042 Rennescedex, France. E-mail: Firstname.Namedirisa.fr

Mohammed Marey is granted by the Egyptian Government becomes of full rank and no redundancy space is left for projecting any constraint. This is a limitation of the classical gradient projection method. That is why a proper selection of the projection operator is required to provide secondary motions of the manipulator that respect the constraints and keep the projected vector from being distorted as much as possible [4],[7]. A nonlinear projection operator has been recently proposed in [2]. We will see that the method we propose leads to significant improvements.

In this paper, we propose a new projection operator. Instead of considering all the $k$ components of the main task e, only the norm $\|\mathbf{e}\|$ of this task is used. Considering the norm of the errors allows the corresponding projection operator to enlarge the permitted motions, at least when the errors are still large. As we will see, using this new projection operator leads to a less constrained problem since the new main task $\|\mathbf{e}\|$ is of rank one at maximum. Our analytical studies show that this operator has to switch to the classical projection operator when the norm of the total error approaches zero. A switching strategy for the projection operator has thus been developed. Finally, an adaptive gain is also proposed to slow down the convergence of the main task. It allows the secondary tasks to be active for longer, which may be useful in practice when the secondary tasks have really to be taken into account (for obstacles or joint limits avoidance for instance).

This paper is organized as follow: in Section II, the new projection operator is developed and discussed. In Section III, different test cases with respect to varying number of task components and robot DOFs are presented and analytically studied as e nears zero. In Section IV, a switching strategy is given to solve the problems exhibited in Sections II and III. Finally, a description of the implementation of the new projection operator followed by experimental results in visual servoing are given in Section VI.

\section{The NeW PROJection operator $\mathbf{P}_{\|\mathbf{e}\|}$}

Let $\mathbf{e} \in \mathbb{R}^{k}$ be the main task function where $\mathrm{k}$ is the number of its components. The classical approach that tries to ensure an exponential decrease of all components of $\mathbf{e}$ leads to the following control scheme [10]:

$$
\begin{aligned}
\dot{\mathbf{q}} & =\dot{\mathbf{q}}_{\mathbf{e}}+\mathbf{P}_{\mathbf{e}} \mathbf{g} \\
& =-\lambda \mathbf{J}_{\mathbf{e}}^{+} \mathbf{e}+\left(\mathbf{I}_{n}-\mathbf{J}_{\mathbf{e}}{ }^{+} \mathbf{J}_{\mathbf{e}}\right) \mathbf{g}
\end{aligned}
$$

where $\dot{\mathbf{q}}$ is the robot joint velocity sent as inputs of the low level robot controller, $\mathbf{J}_{\mathbf{e}} \in \mathbb{R}^{k \times n}$ is the task Jacobian defined such that $\dot{\mathbf{e}}=\mathbf{J}_{\mathrm{e}} \dot{\mathbf{q}}, \mathrm{n}$ is the number of robot DOFs, $\mathbf{J}_{\mathbf{e}}^{+}$is the Moore-Penrose pseudoinverse of $\mathbf{J}_{\mathbf{e}}$, $\mathbf{g}$ represents the motion induced by the secondary task, and 
$\mathbf{P}_{\mathbf{e}}=\left(\mathbf{I}_{n}-\mathbf{J}_{\mathbf{e}}^{+} \mathbf{J}_{\mathbf{e}}\right)$ is a projection operator on the null space of $\mathbf{J}_{\mathbf{e}}$ so that $\mathbf{g}$ is realized at best under the constraint that it does not perturb the regulation of $\mathbf{e}$ to $\mathbf{0}$. This projection operator may be too much constraining: it has no component available when the error e constrains all the $n$ DOFs of the system, and only $n-r$ (where $r$ is the rank of $\mathbf{J}_{\mathbf{e}}$ ) in the general case.

The main original idea of this paper is to define the main task function as $\eta=\|\mathbf{e}\|$. We can then deduce the analytical form of the new projection operator $\mathbf{P}_{\|\mathbf{e}\|}$ and the articular velocity $\dot{\mathbf{q}}_{\|\mathbf{e}\|}$. Since the error norm and the error vector are linked by $\|\mathbf{e}\|^{2}=\mathbf{e}^{\top} \mathbf{e}$, we have $2\|\mathbf{e}\|\|\dot{\mathbf{e}}\|=2 \mathbf{e}^{\top} \dot{\mathbf{e}}$, from which we obtain:

$$
\dot{\eta}=\|\dot{\mathbf{e}}\|=\frac{1}{\|\mathbf{e}\|} \quad \mathbf{e}^{\top} \dot{\mathbf{e}}
$$

Since $\dot{\mathbf{e}}=\mathbf{J}_{\mathbf{e}} \dot{\mathbf{q}}$, we obtain by injecting $\dot{\mathbf{e}}$ in (3) :

$$
\dot{\eta}=\frac{1}{\|\mathbf{e}\|} \mathbf{e}^{\top} \mathbf{J}_{\mathbf{e}} \dot{\mathbf{q}}
$$

from which we deduce

$$
\mathbf{J}_{\|\mathbf{e}\|}=\frac{1}{\|\mathbf{e}\|} \quad \mathbf{e}^{\top} \mathbf{J}_{\mathbf{e}}
$$

Note that $\mathbf{J}_{\|\mathbf{e}\|} \in \mathbb{R}^{1 \times n}$ is at most of rank 1. For all e such that $\|\mathbf{e}\| \neq \mathbf{0}$, we then obtain

$$
\mathbf{J}_{\|\mathbf{e}\|}^{+}=\frac{\|\mathbf{e}\|}{\mathbf{e}^{\top} \mathbf{J}_{\mathbf{e}} \mathbf{J}_{\mathbf{e}}^{\top} \mathbf{e}} \quad \mathbf{J}_{\mathbf{e}}^{\top} \mathbf{e}
$$

If we want $\eta$ to have an exponential decrease, i.e. $\dot{\eta}=-\lambda \eta$, then the least square solution $\dot{\mathbf{q}}_{\|\mathbf{e}\|}$ of $\mathbf{J}_{\|\mathbf{e}\|} \dot{\mathbf{q}}_{\|\mathbf{e}\|}=-\lambda \eta$ is given by:

$$
\dot{\mathbf{q}}_{\|\mathbf{e}\|}=-\lambda\|\mathbf{e}\| \mathbf{J}_{\|\mathbf{e}\|}^{+}
$$

and the general control law will be:

$$
\dot{\mathbf{q}}=\dot{\mathbf{q}}_{\|\mathbf{e}\|}+\dot{\mathbf{q}}_{\|\mathbf{e}\|}^{\perp}=\dot{\mathbf{q}}_{\|\mathbf{e}\|}+\mathbf{P}_{\|\mathbf{e}\| \mathbf{g}}
$$

where $\mathbf{P}_{\|\mathbf{e}\|}=\left(\mathbf{I}_{n}-\mathbf{J}_{\|\mathbf{e}\|}{ }^{+} \mathbf{J}_{\|\mathbf{e}\|}\right)$ is a projection operator on the null space of $\mathbf{J}_{\|\mathbf{e}\|}$ and $\mathbf{g}$ is any vector that can be designed to try to realize secondary tasks.

Using (5) and (6), we directly get:

$$
\mathbf{P}_{\|\mathbf{e}\|}=\mathbf{I}_{n}-\frac{1}{\mathbf{e}^{\top} \mathbf{J}_{\mathbf{e}} \mathbf{J}_{\mathbf{e}}^{\top} \mathbf{e}} \mathbf{J}_{\mathbf{e}}^{\top} \mathbf{e} \mathbf{e}^{\top} \mathbf{J}_{\mathbf{e}}
$$

Since $\mathbf{J}_{\|\mathbf{e}\|}$ is at most of full rank 1, we note that $\mathbf{P}_{\|\mathbf{e}\|}$ is at least of rank $n-1$, which will thus not filter a lot the secondary task $\mathrm{g}$. That is the main idea of this work especially if we remember that, in the classical approach, the rank of $\mathbf{P}_{\mathbf{e}}$ is equal to $n-r$. As soon as $r>1$, supplementary directions of motions are thus available to achieve the secondary tasks. That is particularly true when $\mathbf{J}_{\mathbf{e}}$ is of full rank $n$, in which case $\mathbf{P}_{\mathbf{e}}=\mathbf{0}$ and no secondary task at all can be considered in that usual case.

Let us now consider $\dot{\mathbf{q}}_{\|\mathbf{e}\|}$. After injecting (6) in (7) we obtain:

$$
\dot{\mathbf{q}}_{\|\mathbf{e}\|}=-\lambda \frac{\|\mathbf{e}\|^{2}}{\mathbf{e}^{\top} \mathbf{J}_{\mathbf{e}} \mathbf{J}_{\mathbf{e}}^{\top} \mathbf{e}} \quad \mathbf{J}_{\mathbf{e}}^{\top} \mathbf{e}
$$

Using control scheme output $\dot{\mathbf{q}}_{\|\mathbf{e}\|}$ given by (10), a singular configuration is obtained if $\mathbf{e} \in \operatorname{Ker}\left(\mathbf{J}^{\top}\right)$. That is clear from (5) directly. This case corresponds to a local minima of the classical control scheme: $\dot{\mathbf{q}}_{\mathbf{e}}=-\lambda \mathbf{J}_{\mathbf{e}}^{+} \mathbf{e}$ (see (1) and (2)), since $\operatorname{Ker}\left(\mathbf{J}_{\mathbf{e}}^{\top}\right)=\operatorname{Ker}\left(\mathbf{J}_{\mathbf{e}}^{+}\right)$. Another singularity occurs when $\mathbf{e} \rightarrow \mathbf{0}$ if the denominator $\mathbf{e}^{\top} \mathbf{J}_{\mathbf{e}} \mathbf{J}_{\mathbf{e}}^{\top} \mathbf{e}$ has a convergence rate to zero faster than that of the nominator. If the denominator and the nominator have the same convergence rate to zero when $\mathbf{e} \rightarrow \mathbf{0}$, then $\lim _{\mathbf{e} \rightarrow \mathbf{0}} \dot{\mathbf{q}}_{\|\mathbf{e}\|}$ is indeterminate and the system will not be stable nor robust with respect to any perturbation. Of course, we obtain the same results by studying the stability analysis of the control scheme (10). Let us consider the candidate Lyapunov function $V(t)=\eta^{2}$. By taking the derivative of $V(t)$ and injecting (4) in the result we obtain:

$$
\begin{aligned}
\dot{V}(t) & =2 \eta \dot{\eta}=2 \frac{\|\mathbf{e}\|}{\|\mathbf{e}\|} \mathbf{e}^{\top} \mathbf{J}_{\mathbf{e}} \dot{\mathbf{q}} \\
& =2 \mathbf{e}^{\top} \mathbf{J}_{\mathbf{e}} \dot{\mathbf{q}} \quad \text { when } \quad \mathbf{e} \neq \mathbf{0}
\end{aligned}
$$

Considering $\mathbf{e} \neq \mathbf{0}$ and injecting (10) in (11), we get:

$$
\begin{aligned}
\dot{V}(t) & =\frac{-2 \lambda\|\mathbf{e}\|^{2}}{\mathbf{e}^{\top} \mathbf{J}_{\mathbf{e}} \mathbf{J}_{\mathbf{e}}^{\top} \mathbf{e}} \mathbf{e}^{\top} \mathbf{J}_{\mathbf{e}} \mathbf{J}_{\mathbf{e}}^{\top} \mathbf{e} \\
& =-2 \lambda\|\mathbf{e}\|^{2} \quad \text { when } \mathbf{J}_{\mathbf{e}}^{\top} \mathbf{e} \neq 0
\end{aligned}
$$

We have $\dot{V}(t)<0$ as soon as $\mathbf{e} \neq \mathbf{0}$ and $\mathbf{e} \notin \operatorname{Ker}\left(\mathbf{J}_{\mathbf{e}}^{\top}\right)$, thus ensuring the local asymptotic stability of the system as in the classical case [8], but when $\mathbf{e}=\mathbf{0}$. Let us note that the same results are obtained considering other main task functions such as $\eta=\sqrt{\|\eta\|}, \eta=\|\eta\|^{2}$, and even $\eta=\|\mathbf{e}\|^{\gamma}$ whatever the value of $\gamma$. We will see how to deal with these problems of singularity and stability in Section V-A.

\section{Analytical study of $\mathbf{P}_{\|\mathbf{e}\|}$}

This section presents an analytical analysis of the projection operator $\mathbf{P}_{\|\mathbf{e}\|}$ given by (9). On one hand, we can note that, as $\mathbf{e} \rightarrow \mathbf{0}$, the value of $\mathbf{P}_{\|\mathbf{e}\|}$ is unstable since the denominator tends to zero. On the other hand, we would like that $\mathbf{P}_{\|\mathbf{e}\|}$ tends to $\mathbf{P}_{\mathbf{e}}$ when $\mathbf{e} \rightarrow \mathbf{0}$. Indeed, when $\mathbf{e} \rightarrow \mathbf{0}$, no perturbation has to be introduced by the secondary tasks on each component of e to preserve its convergence and stability. In the following, we illustrate this point by two examples.

\section{A. Case when $k=2$ and $n=2$}

If we consider a system with two DOFs and a task with two components $\mathbf{e}=(x, y)$ then $\mathbf{J}_{\mathbf{e}} \in \mathbb{R}^{2 \times 2}$. By assuming that $\mathbf{J}_{\mathbf{e}}$ is of full rank and is given by:

$$
\mathbf{J}_{\mathbf{e}}=\left[\begin{array}{ll}
a & b \\
c & d
\end{array}\right]
$$

we get:

$$
\mathbf{J}_{\mathbf{e}}^{\top} \mathbf{e} \mathbf{e}^{\top} \mathbf{J}_{\mathbf{e}}=\left[\begin{array}{ll}
A^{2} & A B \\
A B & B^{2}
\end{array}\right]
$$

and

$$
\mathbf{e}^{\top} \mathbf{J}_{\mathbf{e}} \mathbf{J}_{\mathbf{e}}^{\top} \mathbf{e}=A^{2}+B^{2}
$$

where $A=(a x+c y)$ and $B=(b x+d y)$. 
By injecting (14) and (15) in (9) and assuming for simplicity that $x=y$ then taking the limit as $\mathbf{e} \rightarrow \mathbf{0}$ for $\mathbf{P}_{\|\mathbf{e}\|}[1,1]$ we get:

$$
\lim _{\mathbf{e} \rightarrow \mathbf{0}} \mathbf{P}_{\|\mathbf{e}\|}[1,1]=1-\frac{(a+c)^{2}}{(a+c)^{2}+(b+d)^{2}} \neq 0
$$

while we have

$$
\mathbf{P}_{\mathbf{e}}=\mathbf{I}_{n}-\mathbf{J}_{\mathbf{e}}^{-1} \mathbf{J}_{\mathbf{e}}=\mathbf{0}
$$

which implies:

$$
\lim _{\mathbf{e} \rightarrow \mathbf{0}} \mathbf{P}_{\|\mathbf{e}\|} \neq \mathbf{P}_{\mathbf{e}}
$$

Similarly, the same result can be obtained for a higher DOFs system when the number of features is equal to the number of the robot DOFs and the task Jacobian is of full rank.

\section{B. Case when $k=2$ and $n=3$}

If a system of three DOFs is considered with the same task $\mathbf{e}=(x, y)$, then the task Jacobian $\mathbf{J}_{\mathbf{e}} \in \mathbb{R}^{2 \times 3}$ is given by:

$$
\mathbf{J}_{\mathbf{e}}=\left[\begin{array}{lll}
a_{1} & a_{2} & a_{3} \\
b_{1} & b_{2} & b_{3}
\end{array}\right]
$$

then we get:

$$
\mathbf{J}_{\mathbf{e}}^{\top} \mathbf{e e}^{\top} \mathbf{J}_{\mathbf{e}}=\left[\begin{array}{ccc}
X^{2} & X Y & X Z \\
X Y & Y^{2} & Y Z \\
X Z & Y Z & Z^{2}
\end{array}\right]
$$

and

$$
\mathbf{e}^{\top} \mathbf{J}_{\mathbf{e}} \mathbf{J}_{\mathbf{e}}^{\top} \mathbf{e}=x^{2} A+2 x y C+y^{2} B
$$

where $X=a_{1} x+b_{1} y, Y=a_{2} x+b_{2} y, Z=a_{3} x+b_{3} y$, $A=\sum_{i=1}^{3} a_{i}^{2}, B=\sum_{i=1}^{3} b_{i}^{2}, C=\sum_{i=1}^{3} a_{i} b_{i}$.

Injecting (20) and (21) in (9) we get:

$$
\mathbf{P}_{\|\mathbf{e}\|}=\left[\begin{array}{ccc}
1-\frac{X^{2}}{D} & \frac{X Y}{D} & \frac{X Z}{D} \\
\frac{X Y}{D} & 1-\frac{Y^{2}}{D} & \frac{Y Z}{D} \\
\frac{X Z}{D} & \frac{Y Z}{D} & 1-\frac{Z^{2}}{D}
\end{array}\right]
$$

where $D=\mathbf{e}^{\top} \mathbf{J}_{\mathbf{e}} \mathbf{J}_{\mathbf{e}}^{\top} \mathbf{e}$. By considering again the particular case $x=y$ and taking the limit of $\mathbf{P}_{\|\mathbf{e}\|}$ when $\mathbf{e} \rightarrow \mathbf{0}$, then multiply the first column of the result by $\mathbf{J}_{\mathbf{e}}$ we get:

$$
\begin{aligned}
\mathbf{J}_{\mathbf{e}} \lim _{\mathbf{e} \rightarrow \mathbf{0}} \mathbf{P}_{\|\mathbf{e}\|}[1,1 . .3] & =\left[\begin{array}{l}
a_{1}-\frac{a_{1} X_{0}^{2}}{D_{0}}+\frac{a_{2} X_{0} Y_{0}}{D_{0}}+\frac{a_{3} X_{0} Z_{0}}{D_{0}} \\
b_{1}-\frac{b_{1} X_{0}^{2}}{D_{0}}+\frac{b_{2} X_{0} Y_{0}}{D_{0}}+\frac{b_{3} X_{0} Z_{0}}{D_{0}}
\end{array}\right] \\
& \neq\left[\begin{array}{l}
0 \\
0
\end{array}\right]
\end{aligned}
$$

where $D_{0}=\sum_{i=1}^{3}\left(a_{i}^{2}+a_{i} b_{i}+b_{i}^{2}\right), X=a_{1}+b_{1}, Y=$ $a_{2}+b_{2}, Z=a_{3}+b_{3}$. This results shows that once again $\lim _{\mathbf{e} \rightarrow \mathbf{0}} \mathbf{P}_{\|\mathbf{e}\|} \neq \mathbf{P}_{\mathbf{e}}$ since we have of course $\mathbf{J}_{\mathbf{e}} \mathbf{P}_{\mathbf{e}}=\mathbf{0}$.

The previous study directs us to the following conclusion: as soon as the system nears its goal (that is when $\mathbf{e} \rightarrow \mathbf{0}$ ) we have to switch $\mathbf{P}_{\|\mathbf{e}\|}$ to the classical projection operator $\mathbf{P}_{\mathbf{e}}$. This switching ensures the convergence of the system, since it allows solving the instability problem of $\mathbf{P}_{\|\mathbf{e}\|}$ as $\mathbf{e} \rightarrow \mathbf{0}$ due to the singularity of $\mathbf{J}_{\|\mathbf{e}\|}$ when $\mathbf{e}=\mathbf{0}$.

\section{SWITCHING BASED PROJECTION OPERATOR}

As discussed before, the new projection operator $\mathbf{P}_{\|\mathbf{e}\|} \nrightarrow$ $\mathbf{P}_{\mathbf{e}}$ as $\mathbf{e} \rightarrow \mathbf{0}$. To ensure that $\mathbf{P}_{\|\mathbf{e}\|} \rightarrow \mathbf{P}_{\mathbf{e}}$, a switching strategy is designed. It consists in defining a convex combination $\mathbf{P}_{\lambda}$ between the classical and the new projection operator such that:

$$
\mathbf{P}_{\lambda}=\bar{\lambda}(\|\mathbf{e}\|) \quad \mathbf{P}_{\|\mathbf{e}\|}+(1-\bar{\lambda}(\|\mathbf{e}\|)) \quad \mathbf{P}_{\mathbf{e}}
$$

where the proposed formula for the switching function $\bar{\lambda}(\|\mathbf{e}\|): \mathbb{R} \rightarrow[0,1]$ is defined by:

$$
\bar{\lambda}(\|\mathbf{e}\|)= \begin{cases}1 & \text { if } e_{1}<\|\mathbf{e}\| \\ \frac{\lambda(\|\mathbf{e}\|)-\lambda_{0}}{\lambda_{1}-\lambda_{0}} & \text { if } e_{0} \leq\|\mathbf{e}\| \leq e_{1} \\ 0 & \text { if }\|\mathbf{e}\|<e_{0}\end{cases}
$$

where $e_{1}$ and $e_{0}$ are two threshold values that define the starting and the ending conditions for the switching period. $\lambda(e): \mathbb{R} \rightarrow \mathbb{R}$ is a continuous monotonically increasing function, such that $\lambda_{1}=\lambda\left(e_{1}\right) \approx 1$ and $\lambda_{0}=\lambda\left(e_{0}\right) \approx 0$. The sigmoid function $\lambda(t)=\frac{1}{1+\exp (-t)}$ shows early exponential growth from zero for negative $t$, which slows to linear growth of slope $1 / 4$ near $t=0$, then approaches one with an exponentially decaying gap for positive $t$ [14]. A good selection for the function $\lambda(\|\mathbf{e}\|)$ is then the sigmoid function given as:

$$
\lambda(\|\mathbf{e}\|)=\frac{1}{1+\exp \left(-12 \frac{\|\mathbf{e}\|-e_{0}}{e_{1}-e_{0}}+6\right)}
$$

where values of $e_{0}$ and $e_{1}$ have to be selected such that the system does not converge too fast during the interval $\left[e_{0}, e_{1}\right]$. This allows the switching to be performed smoothly during a sufficient number of iterations. Figure (1) shows the shape of the switching function $\bar{\lambda}(\|\mathbf{e}\|)$ when $e_{0}=0.1$ and $e_{1}=(0.3,0.5,0.7,0.9)$.

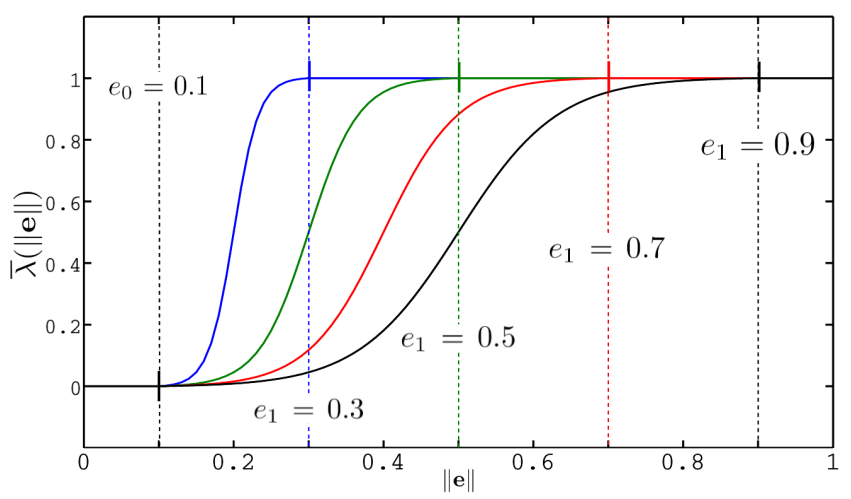

Fig. 1. Switching function $\bar{\lambda}(\|\mathbf{e}\|)$.

\section{MAIN TASK FUNCTION}

\section{A. Possible control schemes}

As discussed before in Section II, stability problem and singularity appear when $\mathbf{e} \rightarrow \mathbf{0}$ if the control scheme $\dot{\mathbf{q}}_{\|\mathbf{e}\|}$ defined in (10) is used as output of the main task. These problems can be avoided by performing a switching from 
$\dot{\mathbf{q}}_{\|\mathbf{e}\|}$ to the classical $\dot{\mathbf{q}}_{\mathbf{e}}$ given in (2) by defining as control scheme:

$$
\dot{\mathbf{q}}_{\lambda}=\bar{\lambda}(\|\mathbf{e}\|) \quad \dot{\mathbf{q}}_{\|\mathbf{e}\|}+(1-\bar{\lambda}(\|\mathbf{e}\|)) \quad \dot{\mathbf{q}}_{\mathbf{e}}
$$

where $\bar{\lambda}(\|\mathbf{e}\|)$ is given by (25) for which $e_{0}$ is selected such that as long as $\|\mathbf{e}\|>e_{0}$, the singularity effect of $\dot{\mathbf{q}}_{\|\mathbf{e}\|}$ does not appear. Applying this switching strategy ensures that the main task will avoid the singularity situation when $\mathbf{e} \rightarrow \mathbf{0}$ since

$$
\lim _{\mathbf{e} \rightarrow \mathbf{0}} \dot{\mathbf{q}}_{\lambda}=\lim _{\mathbf{e} \rightarrow \mathbf{0}} \dot{\mathbf{q}}_{\mathbf{e}}=\mathbf{0}
$$

In order to avoid the indetermination problem when the denominator of $\dot{\mathbf{q}}_{\|\mathbf{e}\|}$ is equal to zero (that is when $\mathbf{e} \in$ $\operatorname{Ker}\left(\mathbf{J}_{\mathbf{e}}^{+}\right)$, a direct switching without a transition interval from $\dot{\mathbf{q}}_{\|\mathbf{e}\|}$ to $\dot{\mathbf{q}}_{e}$ can be employed by setting $e_{0}=e$, where $e$ is the value of the error norm when the indetermination problem occurs. Finally, the classical control scheme $\dot{\mathbf{q}}_{\mathbf{e}}$ can also be used directly with the new projection operator $\mathbf{P}_{\lambda}$ instead of $\dot{\mathbf{q}}_{\|\mathbf{e}\|}$ or $\dot{\mathbf{q}}_{\lambda}$. In that case, the control scheme will be:

$$
\dot{\mathbf{q}}=\dot{\mathbf{q}}_{\mathbf{e}}+\mathbf{P}_{\lambda} \mathbf{g}
$$

Starting the task with the classical control scheme ensures an exponential decreasing of each error component, which may be useful in practice as will be seen in Section VI.

\section{B. Adaptive gain $\beta(\|\mathbf{e}\|)$}

Usually, the gain $\lambda$ involved in the classical control scheme $\dot{\mathbf{q}}_{\mathbf{e}}=-\lambda \mathbf{J}_{\mathbf{e}}^{+} \mathbf{e}$ is tuned so that the convergence rate of the main task is as fast as possible while preserving the stability of the system. This leads to increase $\lambda$ as $\mathbf{e}$ decreases (using for instance $\lambda(\|\mathbf{e}\|)=\lambda_{\text {min }}+K \exp (B\|\mathbf{e}\|)$ where $\lambda_{\min }, K$ and $B$ are constant scalar values). However, having a fast convergence rate for the main task may not be adequate in our case. Indeed, since $\mathbf{P}_{\|\mathbf{e}\|}$ switches to $\mathbf{P}_{\mathbf{e}}$ when $\mathbf{e} \rightarrow \mathbf{0}$, this switch may occur too early to have enough time to take the secondary tasks into account. This is especially the case when the secondary tasks are critical (such as obstacles and joints limits avoidance for example). That is why it may be useful to slow down the convergence of the main task. For that, the norm of the total error is used in the control scheme and we propose to use $\lambda=\lambda_{0}\|\mathbf{e}\|$ where $\lambda_{0}$ is a constant. However, as soon as $\|\mathbf{e}\| \rightarrow 0$, the convergence rate of the main task becomes too small. To avoid this problem, we define a gain function $\beta(\|\mathbf{e}\|)$ which returns the norm of the total error as long as $\mathbf{P}_{\lambda}=\mathbf{P}_{\|\mathbf{e}\|}$ and switches automatically to 1 as soon as the norm of the error reaches a specified threshold value. The scheme of the adaptive gain function $\beta(\|\mathbf{e}\|)$ can be deduced and written as:

$$
\beta(\|\mathbf{e}\|)=1-\bar{\lambda}(\|\mathbf{e}\|)+\|\mathbf{e}\| \bar{\lambda}(\|\mathbf{e}\|)
$$

with the same switching conditions used to switch from $\mathbf{P}_{\|\mathbf{e}\|}$ to $\mathbf{P}_{e}$ used for $\beta(\|\mathbf{e}\|)$. Setting $\lambda=\lambda_{0} \beta(\|\mathbf{e}\|)$, the control scheme (29) becomes:

$$
\dot{\mathbf{q}}=-\lambda_{0} \beta(\|\mathbf{e}\|) \mathbf{J}^{+} \mathbf{e}+\mathbf{P}_{\lambda} \mathbf{g}
$$

Using this control scheme increases the time during which the secondary tasks will be active thanks to the use of $\mathbf{P}_{\|\mathbf{e}\|}$.

\section{EXPERIMENTAL RESUlts}

Results presented in this section have been obtained after applying the proposed methods in visual servoing on a six DOFs robot. In visual servoing [11], the task function is defined by $\mathbf{e}=\mathbf{s}-\mathbf{s}^{*}$ where $\mathbf{s}$ and $\mathbf{s}^{*} \in \mathbb{R}^{k}$ are two vectors representing the current and the desired selected visual features. The task Jacobian $\mathbf{J}_{\mathbf{e}}=\mathbf{L}_{\mathbf{s}} \mathbf{M} \mathbf{J}_{\mathbf{q}}$ where $\mathbf{L}_{\mathbf{s}}$ is the interaction matrix that relates $\dot{\mathbf{s}}$ to the instantaneous camera velocity $\mathbf{v}$ by $\dot{\mathbf{s}}=\mathbf{L}_{\mathbf{s}} \mathbf{v}, \mathbf{J}_{\mathbf{q}}$ is the robot Jacobian and $\mathbf{M}$ is the matrix that relates $\mathbf{v}$ to the variation of the camera pose $\mathbf{p}$ by $\mathbf{v}=\mathbf{M} \dot{\mathbf{p}}$. An object composed of four points forming a square is considered with the Cartesian coordinates of the four points in the image defining the visual features used in the visual servoing system. This means that we have a system of six DOFs and eight visual features with a full rank main task. The camera pose is $\mathbf{p}=(\mathbf{t}, \mathbf{r})$ where $\mathbf{t}$ is a translational vector expressed in meter and $\mathbf{r}$ is the rotational vector expressed in degree. Three different experiments have been implemented to validate and investigate the efficiency of the new projection operator. In all cases, the desired camera pose is $(0,0,0.5,0,0,0)$, which means that the camera has to be at $0.5 \mathrm{~m}$ in front of the square so that it appears as a centered square in the image.

\section{A. Case 1: Control schemes $\dot{\mathbf{q}}_{\|\mathbf{e}\|}$ and $\dot{\mathbf{q}}_{e}$}

In this case, we study the behavior of $\dot{\mathbf{q}}_{\|\mathbf{e}\|}$ and the effect of the adaptive gain $\beta(\|\mathbf{e}\|)$ with the classical control scheme $\dot{\mathbf{q}}_{\mathrm{e}}$. The initial camera pose is $\mathrm{I}=(-0.1,0.1,1.0,0,0,0)$ and no secondary task is added to the main task. Applying $\dot{\mathbf{q}}_{\|\mathrm{e}\|}$, the initial movement consists of translations along $\mathrm{x}-$ axis and y-axis combined with a small rotation around $y-$ axis till iteration number 80, as depicted in Fig. 2(a). Then the translational movement along $\mathrm{z}$-axis starts to increase. Also, Fig. 2(a) shows that as soon as $\|\mathbf{e}\|$ nears zero, the control low $\dot{\mathbf{q}}_{\|\mathbf{e}\|}$ is completely unstable, as explained in Section II. As expected, the norm of the total error is exponentially decreasing but during the instability due to the perturbations it induces. If the switching to $\dot{\mathbf{q}}_{e}$ is performed when $\|\mathbf{e}\| \rightarrow \mathbf{0}$, that is using (27), the system converges as can be seen in Fig. 2(b). As shown in Fig. 2(c), applying the classical control scheme $\dot{\mathbf{q}}_{\mathbf{e}}$, each error component converges exponentially to zero, as well as the norm of the error. Setting the gain $\lambda=\lambda_{0}\|\mathbf{e}\|$, the convergence rate is extremely slow, as depicted in Fig. 2(d). Finally, using the adaptive gain function $\beta(\|\mathbf{e}\|)$ with the classical control law ensures the convergence of the system as shown in Fig. 2(e).

\section{B. Case 2: Secondary task $g=(0.1,0,0,0,0,0)$}

Now, we use the new projection operator and apply a simple secondary task consisting of a translation of $10 \mathrm{~cm} / \mathrm{s}$ along x-axis. Let us first recall that using $\mathbf{P}_{\mathbf{e}}$ would not allow any secondary task to be achieved. As expected, using the projection operator $\mathbf{P}_{\|\mathbf{e}\|}$ during all the servo does not produces a satisfactory behavior (see Fig. 3(a)). Indeed, some secondary motions are produced while the main task tries the robot to reach the desired pose, where it has then to be motionless. This explains the oscillating behavior on the 

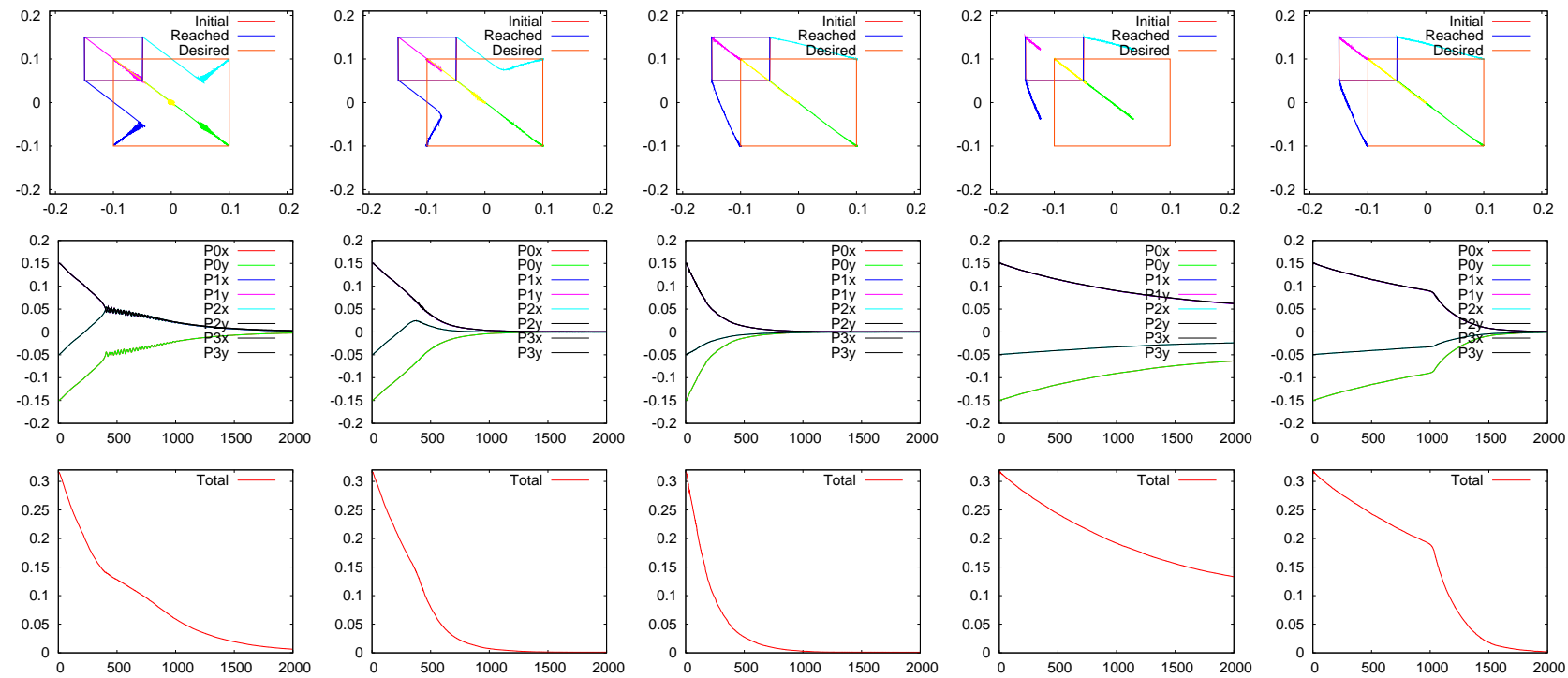

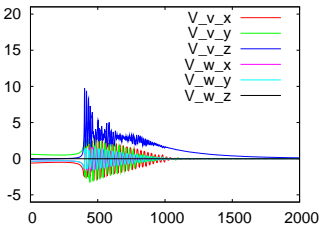

(a) $\dot{\mathbf{q}}_{\|\mathbf{e}\|}$

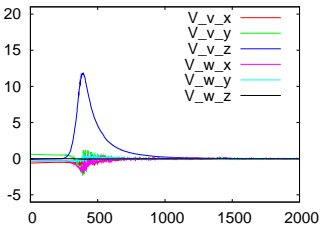

(b) $\dot{\mathbf{q}}_{\|\mathbf{e}\|}$ \& switching

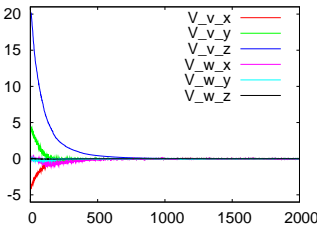

(c) $\dot{\mathbf{q}}_{\mathbf{c}}, \lambda=\lambda_{0}$

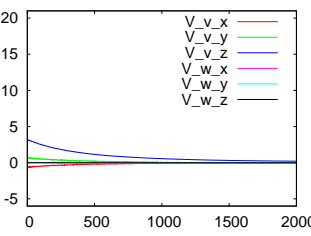

(d) $\dot{\mathbf{q}}_{\mathbf{c}}, \lambda=\lambda_{0}\|\mathbf{e}\|$

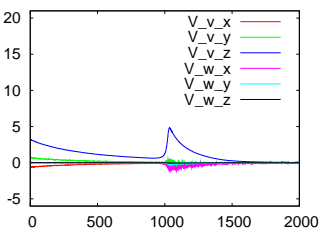

(e) $\dot{\mathbf{q}}_{\mathbf{c}}, \lambda=\lambda_{0} \beta(\|\mathbf{e}\|)$

Fig. 2. Results for case 1. Comparison between the different control schemes Line 1:image points trajectories, line 2: image point error, line 3: norm of the total error, line 4: translational camera velocity $(\mathrm{cm} / \mathrm{s})$ and rotational camera velocity $(\mathrm{deg} / \mathrm{s})$.

velocity components of the global task. In Fig 3(b), the projection operator $\mathbf{P}_{\lambda}$ is used and the system switches automatically to the classical projection operator. This allows the secondary task to be taken into account at the beginning of the servo and ensures the convergence of the system to the desired position. Fig. 3(c) shows the results obtained when the switching gain function $\beta(\|\mathbf{e}\|)$ is used. It is clear that the secondary task is considered during a longer number of iterations, which may be useful in practice.

\section{Case 3: Secondary task $g=(-0.02,0.04,0.02,4,4,4)$}

In this case, a more general secondary task is used with non-zero value in all its components, $g=$ $(-0.02,0.04,0.02,4,4,4)$. Using the new projection operator $\mathbf{P}_{\lambda}$ with the classical control scheme, most of the secondary task components are projected successfully onto the main task, see Fig.3(d). At each iteration, the main task tries keeping the exponential decreasing of each error component while the projection operator tries to keep the exponential decreasing of the norm of the total error, which leads to a nice behavior for the system. Then, thanks to the switching strategy to the classical projection operator, the system converges to the desired pose. When the switching gain function $\beta(\|\mathbf{e}\|)$ is used (see Fig. 3(e)), the number of iterations where the secondary task is considered increases significantly.

\section{CONCLUSION AND FUTURE WORK}

In this paper we have proposed a new large projection operator by defining the main task as the norm of the total error. A switching strategy has been proposed to ensure that the new projection operator smoothly switches to the classical projection operator as soon as the error nears zero. An adaptive gain has also been developed so that the secondary task is effective during a long period by slowing down the convergence rate of the main task. That may be useful when the secondary tasks are extremely important and have really to be considered (which is the case for instance for joints limits and obstacle avoidance).

These theoretical developments have been implemented and validated experimentally on a six DOFs robot arm. The main interest of the new projection operator is that it is always at least of rank (n-1). Hence it can be used even if the main task is of full rank. This property enlarges the applicability of the redundancy framework. As future works, we plan to apply the new projection operator to classical constraints such as joint limits avoidance, obstacle avoidance, occlusion avoidance and also to ensure the visibility of the visual features.

\section{REFERENCES}

[1] B. Nelson and P. Khosla, "Strategies for increasing the tracking region of an eye-in-hand system by singularity and joint limits avoidance", Int. Journal of Robotics Research, 14(3):255-269, June 1995. 

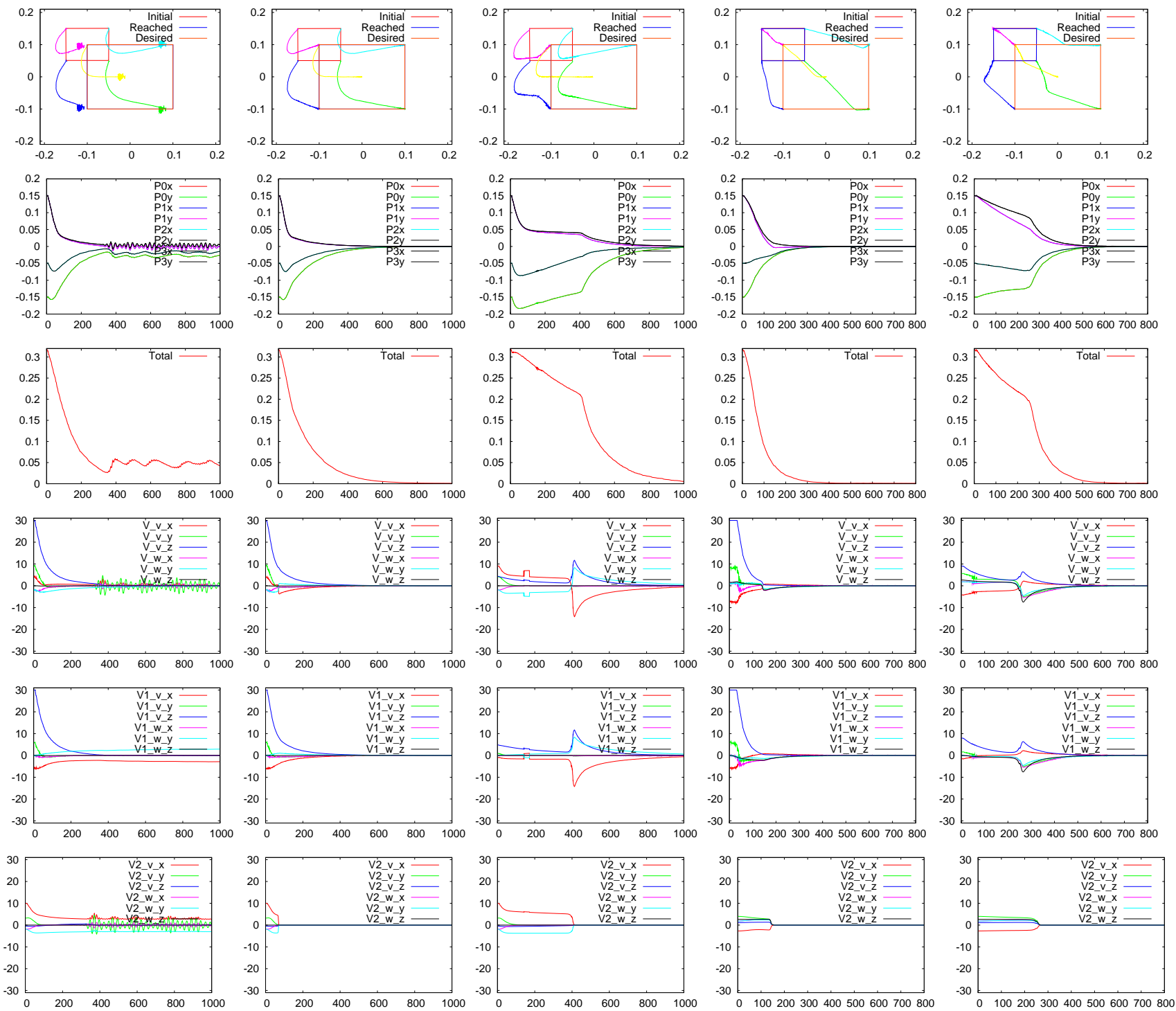

(a) $\dot{\mathbf{q}} \mathbf{e}, \mathbf{P}_{\|\mathbf{e}\|}$

(b) $\dot{\mathbf{q}}_{\mathbf{e}}, P_{\lambda}, \lambda=\lambda_{0}$

(c) $\dot{\mathbf{q}}_{\mathbf{e}}, P_{\lambda}, \lambda=\lambda_{0} \beta(\|\mathbf{e}\|)$

(d) $\dot{\mathbf{q}} \mathbf{e}, P_{\lambda}, \lambda=\lambda_{0}$

(e) $\dot{\mathbf{q}} \mathbf{e}, P_{\lambda}, \lambda=\lambda_{0} \beta(\|\mathbf{e}\|)$

Fig. 3. Results for case 2 are in (a), (b) and (c) and for case 3 in (d) and (f). Line 1: image points trajectories, line 2: image point error, line 3: norm of the total error, lines (4), (5) and (6): camera velocity components in $\mathrm{cm} / \mathrm{s}$ and deg/s of the general, main and secondary tasks respectively.

[2] N. Mansard and F. Chaumette, "Directionnal redundancy for robot control", IEEE Trans. on Automatic Control, 54(6):1179-7192, june 2009

[3] H. Hanafusa, T.Yoshikawa, and Y. Nakamura, "Analysis and control of articulated robot with redundancy", Proc. IFAC, 8th Triennal World Congress, vol. 4, pp. 1927-1932, Kyoto, Japan, 1981.

[4] O. Khatib, "The impact of redundancy on the dynamic performance of robots", Laboratory Robotics and Automation, 8:37-48, 1996.

[5] C. Samson, M. Le Borgne, and B. Espiau, "Robot Control: the Task Function Approach", Clarendon Press, Oxford, England. Oxford Engineering Science Series, 1991

[6] F. Chaumette and E. Marchand, "A redundancy-based iterative scheme for avoiding joint limits: Application to visual servoing", IEEE Trans. on Robotic and Automation., 17(5):719, 2001.

[7] T. Yoshikawa, "Basic optimization methods of redundant manipulators" Laboratory Robotics and Automation. 8:1, 49-60, 1996.

[8] F. Chaumette and S. Hutchinson, "Visual servo control. I. Basic approaches" IEEE Robotics and Automation Magazine, 13(4), Page(s):82 - 90, Dec. 2006.
[9] H Zghal, R V Dubey, and J A Euler, "Efficient gradient projechon optimization lor manipulators with multiple degrees of redundancy", Proc IEEE Int. Conf Robotics and Automation Vol(2):1006-1011, 1990.

[10] A. Liegeois, "Automatic supervisory control of the configuration and behavior of multibody mechanisms", IEEE Trans. on Systems, Man and Cybernetics, 7(12):868-871, Dec. 1977.

[11] B. Espiau, F. Chaumette, P. Rives, "A new approach to visual servoing in robotics", IEEE Trans. on Robotics and Automation 8(3):313-326, June 1992.

[12] J. Lenarcic, "Effective secondary task execution of redundant manipulators", Robotica 16(4):457-462, 1998.

[13] B. Siciliano and J-J. Slotine. "A general framework for managing multiple tasks in highly redundant robotic systems", Proc IEEE Int. Conf Robotics and Automation pp. 12111216, 1991.

[14] R. Pearl and L. Reed. "On the rate of growth of the population of the united state since 1790 and its mathematical representation", Proceedings of the National Academy of Sciences , 6(6):page(s)275288, June 1920 . 